\title{
Editorial Overview: Immune Senescence
}

\author{
Ilhem Messaoudi ${ }^{1,2}$
}

\author{
${ }^{1}$ Vaccine and Gene Therapy Institute, Oregon Health and Science University, USA \\ ${ }^{2}$ Division of Pathobiology and Immunology, Oregon National Primate Research Center 505 NW $185^{\text {th }}$ Avenue Beaver- \\ ton, OR 97006, USA
}

Advanced age is associated with a decline in immune fitness commonly referred as "immune senescence". Despite considerable advances in the medical field, infections continue to seriously compromise quality of life of the elderly. Indeed, infectious diseases such as tuberculosis, pneumonia and influenza remain amongst the 10 leading causes of mortality amongst the elderly in the US. Given that the World Health Organization predicts that by 2050, 2 billion people will be at least 60 years of age (http://www.who.int/ageing/ en/), there is an urgent need to better understand the causes and consequences of immune senescence. The articles in this special issue of Open Longevity Sciences provide an excellent overview of the age-related changes in both the adaptive and the innate branches of the immune system, as well as the impact of these on the host response to infectious agents. This issue also examines the impact of neuroendocrine changes on immune senescence and reviews the current field of immune restorative approaches in the elderly.

The most dramatic hallmark of immune senescence is the loss of naïve $\mathrm{T}$ cells and the accumulation of highly differentiated CD8+ memory $\mathrm{T}$ cells that are often oligoclonal. The review by Dr. Woodland and colleagues postulates that both the naïve and memory CD8+ T cell compartments become dysregulated with aging due to decreased thymic output; the accumulation of cross-reactive memory $\mathrm{T}$ cells that can mediate inappropriate inflammatory responses; and the appearance of $\mathrm{T}$ cell clonal expansions, which further compromise the diversity of the $\mathrm{T}$ cell repertoire. The authors present evidence that these events ultimately lead to the failure of the $\mathrm{T}$ cell compartment to mount effective immune responses to both new and previously encountered pathogens.

The accumulation of terminally differentiated $\mathrm{T}$ cell clonal populations has been largely attributed to cytomegalovirus (CMV) infection. More specifically, it is believed that over time, CMV-specific T cell populations become "inflated", usurping a large space within the T cell niche. However, the evidence that CMV infection contributes to the functional failings of the immune system in old age, rather than providing a convenient biomarker, is a highly debated topic. The review by Drs. Hill and Tatum assesses the lit

*Address correspondence to this author at the Division of Pathobiology and Immunology, Oregon National Primate Research Center 505 NW 185 Avenue Beaverton, OR 97006, USA; Tel: 503-418-2734;

Fax: 503-418-2701;E-mail: messaoud@ohsu.edu erature concerning CMV seropositivity and severity of immune senescence and examines the evidence supporting a role for CMV as a driver of altered $\mathrm{T}$ cell homeostasis. The authors present the alternative theory that poor aging might result in decreased control of CMV infection and increased viral burden leading to disturbances in the $\mathrm{T}$ cell repertoire.

Drs. Haynes and Lefebvre review the qualitative and quantitative changes in the CD4+ $\mathrm{T}$ cell compartment that arise with aging, since CD4 $\mathrm{T}$ cells play a central role in the humoral response by providing help to B cells. The authors also summarize the age-related intrinsic and extrinsic factors that impact naïve, memory, and regulatory CD4+ $\mathrm{T}$ cell functions. These studies are critical since a better understanding of the age-related defects in CD4 T cell function will have far-reaching ramifications for improving both the humoral as well as the cellular immune response to infection and vaccination in the elderly.

Dr. Blomberg and colleagues examine defects in B cell function that underlie reduced responses to pneumococcal and influenza vaccines in the elderly. In addition to agerelated defect in T cell help, the authors emphasize that autonomous B cell defects, such as those observed during isotype class switching, also account for reduced responses to vaccines in the elderly. Their article highlights the need for further investigations into the mechanistic basis for agerelated changes in pneumococcal responses, particularly with regards to the function of B1 B cells, which have been reported to decrease with age. Dr. Cancro and colleagues also examine age-related changes in the $\mathrm{B}$ cell repertoire and summarize recent progress in understanding a recently discovered B cell subset in aged mice. This phenotypically and functionally unique subset of mature B cells, termed ageassociated B cells (ABCs), responds poorly to adaptive and most innate stimuli, and is believed to foster aberrant responses by secreting inflammatory and/or negative regulatory cytokines. The authors hypothesize that the accumulation of the ABC subset may bias the immune system toward inflammatory or autoimmune responses, and/or impair the generation of classical plasma and memory B cells.

Immune senescence also affects the innate immune system. The review by Dr. Fulop and colleagues describes the changes in the innate immune response with aging. They illustrate age-related changes in neutrophil homeostasis, phagocytosis, chemotaxis, and intracellular killing. In addition, the authors describe changes in the functional responses of macrophages and dendritic cells to TLR stimulation as 
evidenced by reduced phagocytosis, chemotaxis, dysregulated cytokine production and lack of co-stimulatory receptor upregulation. The review also examines changes in NK cells, describing alterations in both NK subset frequency and function with age.

These age-related disturbances in innate and adaptive immunity result in increased susceptibility to infection in the elderly. This is highlighted in the article by Dr. Turner and colleagues, who examine the mechanisms underlying increased vulnerability of the elderly to mycobacterium tuberculosis (M.tb) infections. It is generally believed that ageassociated changes in immune function, and more specifically $\mathrm{T}$ cell senescence, result in the reactivation of latent M.tb infection, the main form of TB in the elderly. However, the contribution of defects in T cell function to the loss of compact granuloma formation and integrity is not well understood. The review also discusses the reduced efficacy of diagnostic tools in the elderly and the increased toxicity of drugs normally used in young patients. Similarly, multiple recent population-based studies document the rising prevalence of lung disease caused by the environmentally ubiquitous nontuberculous mycobacteria (NTM), particularly among older women. This is the focus of the article by Drs. McNelly and Winthrop who review clinical features of NTM lung disease, host response, immunological deficiencies that pre-dispose the elderly to this disease, and potential factors that contribute to the gender bias observed. Amongst the many predisposing conditions for NTM lung disease that have been identified to date, chronic lung disease and estrogen deprivation among post-menopausal women seem to play a critical role. The impact of estrogen deprivation is discussed in more detail in the review by Dr. Messaoudi and colleagues, which explores the link between age-related loss of ovarian steroids and immune senescence in women. Several studies demonstrated that ovarian steroids modulate immune response to vaccination and infection in women, but the impact of menopause on immune function remains poorly understood. This review examines several lines of evi- dence that suggest that loss of ovarian sex steroids impact inflammatory cytokine levels as well as the frequency and function of immune cells in post-menopausal women.

The increased susceptibility of the elderly to infection is further compounded by decreased responses to vaccinations. Dr. Amana reviews the impact of immune senescence on the immune response following immunization in the elderly. This review reminds us that some vaccines may pose a substantial safety risk in the elderly when compared to younger individuals. The review also explores several domestic and travel vaccines, examining what is known concerning their efficacy and safety in the elderly. Finally, the review considers future alternatives such as increased vaccine dosages, new adjuvants, and novel vaccine antigens, which may provide safer and more efficacious vaccines for seniors in the future.

Dr. Govind and colleagues examine the question of immune restorative interventions in the elderly. Given that immune senescence does not describe a uniform decline in immune function, the authors discuss who might best benefit from such immunotherapies, how they might be treated, and when to start the treatment. Their review emphasized the complexity of "immune senescence" and the need for approaches that can better identify and measure it. They also highlight the need for additional large-scale clinical studies to identify improved biomarkers of immune senescence, and that future immune rejuvenation approaches should include multi-pronged approaches.

In summary, the authors of the papers contained herein have provided an outstanding overview of the many facets of immune senescence and its impact on the response to infection and vaccination. In many cases, potential interventions to improve immunity in the elderly were discussed. We hope that increased understanding of the immunological changes with age can lead to novel targeted approaches to alleviate morbidity and mortality from infectious diseases in the elderly.

(C) Ilhem Messaoudi; Licensee Bentham Open.

This is an open access article licensed under the terms of the Creative Commons Attribution Non-Commercial License (http://creativecommons.org/licenses/by-nc/3.0/) which permits unrestricted, non-commercial use, distribution and reproduction in any medium, provided the work is properly cited. 